JOURNAL OF THE

CHUNGCHEONG MATHEMATICAL SOCIETY

Volume 27, No. 2, May 2014

http://dx.doi.org/10.14403/jcms.2014.27.2.165

\title{
RADO'S AND POPONOV'S INEQUALITIES OF PROBABILITY MEASURES FOR POSITIVE REAL NUMBERS
}

\author{
Hosoo LeE* And Sejong KIm**
}

\begin{abstract}
In this paper, we derive some valuable inequalities of Rado's and Poponov's types on the open interval of positive real numbers, and then show weighted generalizations of Rado's and Poponov's inequalities on the set of positive real numbers equipped with compactly supported probability measure.
\end{abstract}

\section{Introduction}

Let $\mathbf{a}=\left(a_{1}, \ldots, a_{n}\right)$ be an $n$-tuple of positive real numbers. The arithmetic and geometric means of $n$ positive real numbers are well known:

$$
A_{n}(\mathbf{a})=\frac{a_{1}+a_{2}+\cdots+a_{n}}{n} \text { and } G_{n}(\mathbf{a})=\sqrt[n]{a_{1} a_{2} \cdots a_{n}},
$$

respectively. The following inequalities

$$
\begin{aligned}
n\left(A_{n}(\mathbf{a})-G_{n}(\mathbf{a})\right) & \geq(n-1)\left(A_{n-1}(\mathbf{a})-G_{n-1}(\mathbf{a})\right), \\
\left(\frac{A_{n}(\mathbf{a})}{G_{n}(\mathbf{a})}\right)^{n} & \geq\left(\frac{A_{n-1}(\mathbf{a})}{G_{n-1}(\mathbf{a})}\right)^{n-1}
\end{aligned}
$$

are known in the literature as Rado's inequality and Poponov's inequality, respectively (see $[2$, p.94]). Inequalities (1.1) and (1.2) provide a good approach for combining arithmetic means and geometric means of positive real numbers. This pair of inequalities has received considerable attention by many authors, and has motivated a large number of papers giving their simple proofs providing various generalizations, improvements and analogues $[2,1,3,5,4]$.

Received October 11, 2013; Accepted January 06, 2014.

2010 Mathematics Subject Classification: Primary 28A25; Secondary 26D07.

Key words and phrases: Rado's inequality, Poponov's inequality, compactly supported measure.

Correspondence should be addressed to Sejong Kim, skim@chungbuk.ac.kr. 
The aim of this paper is to establish a new generalization of Rado's and Poponov's inequalities which involves probability measure with compact support.

\section{Compactly supported measures}

In this section we generally see certain type of probability measure, namely compactly supported measure. Let $X$ be a topological space and $f: X \rightarrow \mathbb{R}$ a function. The support of $f$, denoted by $\operatorname{supp}(f)$, is the smallest closed set outside of which the function $f$ vanishes identically. If the function $f$ vanishes outside a compact set $C$, we say that $f$ has compact support (or that $f$ is the function of compact support). For the case that $f=\mu$, a probability measure, we denote as $\mathcal{P}$ and $\mathcal{P}_{c}$ a set of all probability measures and a set of probability measures of compact support, respectively, on the topological space $X$.

The Borel $\sigma$-algebra $\mathcal{B}(X)$ of the topological space $X$ is the smallest $\sigma$-algebra containing all open subsets of $X$. We call $(X, \mathcal{B}(X), \mu)$ a measure space and simply denote by $(X, \mu)$. In the following we show that any continuous function from $(X, \mu)$ with $\mu \in \mathcal{P}_{c}$ to a Banach space is Bochner $\mu$-integrable.

Lemma 2.1. Every continuous function $f$ from $(X, \mu)$ with $\mu \in \mathcal{P}_{c}$ to a Banach space is strongly $\mu$-measurable, and furthermore, Bochner $\mu$-integrable.

Proof. Let $n$ be a positive integer and $x \in X$. We define an open set

$$
O_{n, x}:=\left\{y \in X:\|f(x)-f(y)\|<\frac{1}{n}\right\} .
$$

Then the collection $\left\{O_{n, x}: x \in X\right\}$ is an open cover of $X$. Since $\operatorname{supp}(\mu)$ is a compact subset of $X$, there exists a finite subcover $\left\{O_{n, x_{i}}: i=\right.$ $\left.1, \ldots, N_{n}\right\}$ of $\operatorname{supp}(\mu)$.

We now construct a sequence of $\mu$-simple functions $f_{n}$ such that

$$
f_{n}=\sum_{i=1}^{N_{n}} f\left(a_{i}\right) 1_{\mathbf{B}_{n, i}},
$$

where $\left\{\mathbf{B}_{n, i}: i=1, \ldots, N_{n}\right\}$ is the partition of $\operatorname{supp}(\mu)$ defined by

$$
\begin{aligned}
& \mathbf{B}_{n, 1}:=O_{n, x_{1}} \cap \operatorname{supp}(\mu), \\
& \mathbf{B}_{n, k}:=\left(O_{n, x_{k}} \backslash \bigcup_{j<k} O_{n, x_{j}}\right) \cap \operatorname{supp}(\mu), 2 \leq k \leq N_{n},
\end{aligned}
$$


and $a_{i}$ is an evaluation point arbitrarily chosen in $\mathbf{B}_{n, i}$. Then for $x \in$ $\mathbf{B}_{n, j} \subset \operatorname{supp}(\mu)$

$\left\|f_{n}(x)-f(x)\right\|=\left\|f\left(a_{j}\right)-f(x)\right\| \leq\left\|f\left(a_{j}\right)-f\left(x_{j}\right)\right\|+\left\|f\left(x_{j}\right)-f(x)\right\|<\frac{2}{n}$.

So $\lim _{n \rightarrow \infty} f_{n}=f$ pointwise on $\operatorname{supp}(\mu)$, and hence $\mu$-almost everywhere. Thus $f$ is strongly $\mu$-measurable. Furthermore,

$$
\begin{aligned}
\int_{X}\left\|f_{n}-f\right\| d \mu=\int_{\operatorname{supp}(\mu)}\left\|f_{n}-f\right\| d \mu & =\sum_{i=1}^{N_{n}} \int_{\mathbf{B}_{n, i}}\left\|f_{n}-f\right\| d \mu \\
& <\sum_{i=1}^{N_{n}} \int_{\mathbf{B}_{n, i}} \frac{2}{n} d \mu=\frac{2}{n},
\end{aligned}
$$

and hence $\lim _{n \rightarrow \infty} \int_{X}\left\|f_{n}-f\right\| d \mu=0$.

REMARK 2.2. We can see from the proof of Lemma 2.1 that a continuous function $f$ from $(X, \mu)$ with $\mu \in \mathcal{P}_{c}$ to a Banach space is a limit of $\mu$-simple functions, each defined from a Borel partition of $\operatorname{supp}(\mu)$. In other words,

$$
\int_{X} f d \mu=\lim _{n \rightarrow \infty} \sum_{i=1}^{N_{n}} f\left(a_{i}\right) \mu\left(\mathbf{B}_{n, i}\right),
$$

where $\left\{\mathbf{B}_{n, i}: i=1, \ldots, N_{n}\right\}$ is a Borel partition of $\operatorname{supp}(\mu)$ and $a_{i}$ is a sample point arbitrarily chosen in $\mathbf{B}_{n, i}$. The integral for the continuous function is independent of the approximating partition for $\operatorname{supp}(\mu)$, as long as $\left\{\mathbf{B}_{n, i}: i=1, \ldots, N_{n}\right\}$ refines $\left\{O_{n, x_{i}}: i=1, \ldots, N_{n}\right\}$.

\section{Power means of positive real numbers}

The power mean on the set of positive real numbers equipped with compactly supported measure $\mu$ is defined by

$$
M^{[p]}(\mu)=\left(\int_{(0, \infty)} x^{p} \mu(d x)\right)^{1 / p}, p \neq 0 .
$$

Note that

$$
M^{[1]}(\mu)=\int_{(0, \infty)} x \mu(d x) \text { and } M^{[-1]}(\mu)=\left(\int_{(0, \infty)} x^{-1} \mu(d x)\right)^{-1}
$$


are known as the arithmetic and harmonic means with respect to $\mu$, and are denoted by $A(\mu)$ and $H(\mu)$, respectively. Furthermore, the limit of $M^{[p]}(\mu)$ as $p \rightarrow 0$ is given by

$$
M^{[0]}(\mu):=\lim _{p \rightarrow 0} M^{[p]}(\mu)=\exp \int_{(0, \infty)} \log x \mu(d x) .
$$

We call it the geometric mean with respect to $\mu$ and denote by $G(\mu)$.

REMARK 3.1. Since the function $f(x)=x^{p}$ is continuous, Lemma 2.1 tells that for any given $k \in \mathbb{N}$, there exists a partition

$$
\mathbb{B}_{k}=\left\{\mathbf{B}_{k, 1}, \ldots, \mathbf{B}_{k, N}\right\}
$$

of $\operatorname{supp}(\mu)$ satisfying

$$
\begin{aligned}
& \sum_{i=1}^{N} x_{i}^{*} \mu\left(\mathbf{B}_{k, i}\right)-\frac{1}{k}<\int_{(0, \infty)} x \mu(d x)<\sum_{i=1}^{N} x_{i}^{*} \mu\left(\mathbf{B}_{k, i}\right)+\frac{1}{k} \\
& \prod_{i=1}^{N}\left(x_{i}^{*}\right)^{\mu\left(\mathbf{B}_{k, i}\right)}-\frac{1}{k}<\exp \int_{(0, \infty)} \log x \mu(d x) \leq \prod_{i=1}^{N}\left(x_{i}^{*}\right)^{\mu\left(\mathbf{B}_{k, i}\right)}+\frac{1}{k}
\end{aligned}
$$

for any sample point $x_{i}^{*} \in B_{k, i}$ such that $x_{i}^{*}>\frac{1}{k}$.

Let $\mathbf{a}=\left(a_{1}, \ldots, a_{n}\right)$ be an $n$-dimensional vector of nonnegative real numbers, not all zero. For an $n$-dimensional probability vector $\omega=$ $\left(w_{1}, \ldots, w_{n}\right)$, we denote the weighted version of arithmetic and geometric means as follows:

$$
A(\omega ; \mathbf{a})=\sum_{i=1}^{n} w_{i} a_{i}, G(\omega ; \mathbf{a})=\prod_{i=1}^{n} a_{i}^{w_{i}} .
$$

One can see that if the measure $\mu$ is the $n$-dimensional probability vector, then $M^{[1]}(\mu)$ and $M^{[0]}(\mu)$ can be written as the above expression. It is known that

$$
G(\omega ; \mathbf{a}) \leq A(\omega ; \mathbf{a})
$$

and the equality holds when $a_{1}=\cdots=a_{n}$.

\section{Rado's inequality}

In this section we prove the extension of Rado's inequality under compactly supported measure with the following lemma. 
Lemma 4.1. Let $\mathbf{a} \in(0, \infty)^{m}, \mathbf{b} \in(0, \infty)^{n}$, and let $\omega=\left(w_{1}, \ldots, w_{m}\right)$ and $\nu=\left(v_{1}, \ldots, v_{n}\right)$ be probability vectors. Then for any $0<t<1$

$$
\begin{aligned}
& (1-t) A(\omega ; \mathbf{a})+t A(\nu ; \mathbf{b})-G(\omega ; \mathbf{a})^{1-t} G(\nu ; \mathbf{b})^{t} \\
& \geq(1-t)[A(\omega ; \mathbf{a})-G(\omega ; \mathbf{a})]+t[A(\nu ; \mathbf{b})-G(\nu . \mathbf{b})]
\end{aligned}
$$

Proof. Note that the inequality (4.1) is equivalent to

$$
G(\omega ; \mathbf{a})^{1-t} G(\nu ; \mathbf{b})^{t} \leq(1-t) G(\omega ; \mathbf{a})+t G(\nu ; \mathbf{b}) .
$$

This is true due to the arithmetic-geometric mean inequality (3.1) of two variables.

TheOREM 4.2. Let $\mu, \nu_{1}, \nu_{2}$ be compactly supported measures on $(0, \infty)$, and let $\mu$ be a linear convex combination of $\nu_{1}$ and $\nu_{2}$, i.e.,

$$
\mu=(1-t) \nu_{1}+t \nu_{2}
$$

for $t \in(0,1)$. Then

$$
A(\mu)-G(\mu) \geq(1-t)\left[A\left(\nu_{1}\right)-G\left(\nu_{1}\right)\right]+t\left[A\left(\nu_{2}\right)-G\left(\nu_{2}\right)\right] .
$$

Proof. By the condition of partition $\mathbb{B}_{k}$ in Remark 3.1 and Lemma 4.1, we have

$$
\begin{aligned}
A(\mu)-G(\mu)+\frac{2}{k} & \left(\int_{(0, \infty)} x \mu(d x)+\frac{1}{k}\right)-\left(\exp \int_{(0, \infty)} \log x \mu(d x)-\frac{1}{k}\right) \\
\geq & \sum_{i=1}^{N} x_{i}^{*} \mu\left(\mathbf{B}_{k, i}\right)-\prod_{i=1}^{N}\left(x_{i}^{*}\right)^{\mu\left(\mathbf{B}_{k, i}\right)} \\
\geq & (1-t)\left[\sum_{i=1}^{N} x_{i}^{*} \nu_{1}\left(\mathbf{B}_{k, i}\right)-\prod_{i=1}^{N}\left(x_{i}^{*}\right)^{\nu_{1}\left(\mathbf{B}_{k, i}\right)}\right] \\
& +t\left[\sum_{i=1}^{N} x_{i}^{*} \nu_{2}\left(\mathbf{B}_{k, i}\right)-\prod_{i=1}^{N}\left(x_{i}^{*}\right)^{\nu_{2}\left(\mathbf{B}_{k, i}\right)}\right] \\
= & (1-t)\left[\left(\sum_{i=1}^{N} x_{i}^{*} \nu_{1}\left(\mathbf{B}_{k, i}\right)+\frac{1}{k}\right)-\left(\prod_{i=1}^{N}\left(x_{i}^{*}\right)^{\nu_{1}\left(B_{k, i}\right)}-\frac{1}{k}\right)\right] \\
& +\left[\left(\sum_{i=1}^{N} x_{i}^{*} \nu_{2}\left(\mathbf{B}_{k, i}\right)+\frac{1}{k}\right)-\left(\prod_{i=1}^{N}\left(x_{i}^{*}\right)^{\nu_{2}\left(\mathbf{B}_{k, i}\right)}-\frac{1}{k}\right)\right]-\frac{2}{k}
\end{aligned}
$$




$$
\begin{aligned}
& \geq(1-t)\left[\int_{(0, \infty)} x \nu_{1}(d x)-\exp \int_{(0, \infty)} \log x \nu_{1}(d x)\right] \\
& \quad+t\left[\int_{(0, \infty)} x \nu_{2}(d x)-\exp \int_{(0, \infty)} \log x \nu_{2}(d x)\right]-\frac{2}{k} \\
& =(1-t)\left[A\left(\nu_{1}\right)-G\left(\nu_{1}\right)\right]+t\left[A\left(\nu_{2}\right)-G\left(\nu_{2}\right)\right]-\frac{2}{k}
\end{aligned}
$$

As $k$ is arbitrary, we have

$$
A(\mu)-G(\mu) \geq(1-t)\left[A\left(\nu_{1}\right)-G\left(\nu_{1}\right)\right]+t\left[A\left(\nu_{2}\right)-G\left(\nu_{2}\right)\right] .
$$

REMARK 4.3. Let us consider the $n$-dimensional probability measures $\mu, \nu_{1}$, and $\nu_{2}$ :

$$
\mu=\left(\frac{1}{n}, \ldots, \frac{1}{n}\right), \nu_{1}=\left(\frac{1}{n-1}, \ldots, \frac{1}{n-1}\right), \nu_{2}=(0, \ldots, 0,1) .
$$

Then $\mu=\left(1-\frac{1}{n}\right) \nu_{1}+\frac{1}{n} \nu_{2}$, and so Theorem 4.2 with $t=1 / n$ yields

$$
A_{n}-G_{n} \geq\left(1-\frac{1}{n}\right)\left(A_{n-1}-G_{n-1}\right) .
$$

Thus we obtain the original Rado's inequality (1.1).

\section{Poponov's inequality}

In this section we prove the extension of Poponov's inequality under compactly supported measure with the following lemmas.

Lemma 5.1. Let $\mathbf{a} \in(0, \infty)^{m}, \mathbf{b} \in(0, \infty)^{n}$, and let $\omega=\left(w_{1}, \ldots, w_{m}\right)$ and $\nu=\left(v_{1}, \ldots, v_{n}\right)$ be probability vectors. Then for any $0<t<1$,

$$
\frac{(1-t) A(\omega ; \mathbf{a})+t A(\nu ; \mathbf{b})}{G(\omega ; \mathbf{a})^{1-t} G(\nu ; \mathbf{b})^{t}} \geq\left(\frac{A(\omega ; \mathbf{a})}{G(\omega ; \mathbf{a})}\right)^{1-t}\left(\frac{A(\nu ; \mathbf{b})}{G(\nu ; \mathbf{b})}\right)^{t} .
$$

Proof. The inequality (5.1) is equivalent to

$$
(1-t) A(\omega ; \mathbf{a})+t A(\nu ; \mathbf{b}) \geq A(\omega ; \mathbf{a})^{1-t} A(\nu ; \mathbf{b})^{t} .
$$

This is true due to the arithmetic-geometric mean inequality (3.1) of two variables.

The proof of the following lemma is straightforward. 
LEMMA 5.2. If $G>\frac{1}{k}$ for some $k \in \mathbb{N}$, then

$$
\frac{A}{G}+C_{k} \geq \frac{A+\frac{1}{k}}{G-\frac{1}{k}}
$$

where $C_{k}=\frac{A+G}{G(k G-1)}$.

THEOREM 5.3. Let $\mu, \nu_{1}, \nu_{2}$ be compactly supported measures on $(0, \infty)$, and let $\mu$ be a linear convex combination of $\nu_{1}$ and $\nu_{2}$. Then for $0<t<1$

$$
\frac{A(\mu)}{G(\mu)} \geq\left[\frac{A\left(\nu_{1}\right)}{G\left(\nu_{1}\right)}\right]^{1-t}\left[\frac{A\left(\nu_{2}\right)}{G\left(\nu_{2}\right)}\right]^{t} .
$$

Proof. By Remark 3.1, Lemma 5.2 is valid. By Lemma 5.1, we have

$$
\begin{aligned}
\frac{A(\mu)}{G(\mu)}+C_{k} & \geq \frac{A(\mu)+\frac{1}{k}}{G(\mu)-\frac{1}{k}} \\
& \geq \frac{\sum_{i=1}^{N} x_{i}^{*} \mu\left(\mathbf{B}_{k, i}\right)}{\prod_{i=1}^{N}\left(x_{i}^{*}\right)^{\mu\left(\mathbf{B}_{k, i}\right)}}=\frac{(1-t) \sum_{i=1}^{N} x_{i}^{*} \nu_{1}\left(\mathbf{B}_{k, i}\right)+t \sum_{i=1}^{N} x_{i}^{*} \nu_{2}\left(\mathbf{B}_{k, i}\right)}{\left[\prod_{i=1}^{N}\left(x_{i}^{*}\right)^{\nu_{1}\left(\mathbf{B}_{k, i}\right)}\right]^{1-t}\left[\prod_{i=1}^{N}\left(x_{i}^{*}\right)^{\nu_{2}\left(\mathbf{B}_{k, i}\right)}\right]^{t}} \\
& \geq\left[\frac{\sum_{i=1}^{N} x_{i}^{*} \nu_{1}\left(\mathbf{B}_{k, i}\right)}{\prod_{i=1}^{N}\left(x_{i}^{*}\right)^{\nu_{1}\left(\mathbf{B}_{k, i}\right)}}\right]^{1-t}\left[\frac{\sum_{i=1}^{N} x_{i}^{*} \nu_{2}\left(\mathbf{B}_{k, i}\right)}{\prod_{i=1}^{N}\left(x_{i}^{*}\right)^{\nu_{2}\left(\mathbf{B}_{k, i}\right)}}\right]^{t} .
\end{aligned}
$$

As $k \rightarrow \infty, C_{k} \rightarrow 0$ and

$$
\frac{A(\mu)}{G(\mu)} \geq\left[\frac{A\left(\nu_{1}\right)}{G\left(\nu_{1}\right)}\right]^{1-t}\left[\frac{A\left(\nu_{2}\right)}{G\left(\nu_{2}\right)}\right]^{t} .
$$

REMARK 5.4. For the $n$-dimensional probability measures $\mu, \nu_{1}$, and $\nu_{2}$ defined in Remark 4.3, Theorem 5.3 with $t=1 / n$ yields

$$
\frac{A_{n}}{G_{n}} \geq\left[\frac{A_{n-1}}{G_{n-1}}\right]^{1-\frac{1}{n}} .
$$


Thus we obtain the original Poponov's inequality $\left[\frac{A_{n}}{G_{n}}\right]^{n} \geq\left[\frac{A_{n-1}}{G_{n-1}}\right]^{n-1}$.

\section{References}

[1] D. S. Mitrinović, J. E. Pecarić, and A. M. Fink, Classical and New Inequalities in Analysis, Kluwer Academic Publishers, Dordrecht, 1993, 2126.

[2] D. S. Mitrinović and P. M. Vasić, Analytic Inequalities, Springer-Verlag, New York, 1970, 7494.

[3] S. Wu, Generalization of Rados inequality involving multiple parameters and its applications, College Math. 21, (2005) 107111.

[4] $\mathrm{S}$. Wu, Generalization and sharpness of power means inequality and their applications, J. Math. Anal. Appl. 312 (2005) 637652.

[5] S. Wu, Two generalized versions of Popovicius inequality, J. Sichuan Normal Univ. Nat. Sci. Ed. 27 (2004), no. 3, 251254.

College of Basic Studies

Yeungnam University

Gyeongsan 712-749, Republic of Korea

E-mail: hosoo@yu.ac.kr

**

Department of Mathematics

Chungbuk National University

Cheongju 361-763, Republic of Korea

E-mail: skim@chungbuk.ac.kr 